\title{
Non-parathyroid hypercalcemia during lactation: a case report
}

\author{
Wei Shi", Xiajuan Wang", Jiayue Yang, Lan Xu, Ruifang Bu \\ Endocrine Department, Wuxi People's Hospital Affiliated to Nanjing Medical University, Wuxi, China \\ \#These authors contributed equally to this work. \\ Correspondence to: Ruifang Bu; Lan Xu. Endocrine Department, Wuxi People's Hospital Affiliated to Nanjing Medical University, No. 299, Qingyang \\ Road, Liangxi District, Wuxi 214000, China. Email: brfang@163.com; xulan126@126.com.
}

\begin{abstract}
Hypercalcemia is a clinical emergency which can cause hypercalcemic crisis and even endanger patients' lives. The increase of serum calcium concentration is caused by the redistribution of calcium in bone and the inhibition of parathyroid secretion, which is known as non-parathyroid hypercalcemia. In this report, we presented a rare case of non-parathyroid hypercalcemia during lactation in order to optimize the diagnosis and treatment of this condition. A 27 -year-old female patient was admitted to Wuxi People's Hospital on July 11, 2019 due to "fatigue, anorexia, and pain in both knees for half a month". The patient had fatigue and discomfort, accompanied by pain in both knees without obvious inducement. At the same time, the patient had decreased food intake. In the past 3 days, the symptoms worsened, accompanied by limb numbness. The serum calcium level was increased and the parathyroid hormone (PTH) level was decreased. The patient was diagnosed with hypercalcemia, and was treated with calcitonin and lactation termination. The knee pain disappeared and serum calcium returned to normal during a 2-week follow-up. To conclude, the correlation between hypercalcemia and lactation needs to be considered for non-parathyroid hypercalcemia during lactation. After excluding other possible causes, lactation termination therapy may be an effective therapeutic strategy for non-parathyroid hypercalcemia caused by excessive lactation.
\end{abstract}

Keywords: Hypercalcemia; lactation; parathyroid hormone (PTH); parathyroid hormone related peptide; case report

Submitted Jun 22, 2021. Accepted for publication Aug 18, 2021.

doi: 10.21037/apm-21-2196

View this article at: https://dx.doi.org/10.21037/apm-21-2196

\section{Introduction}

The maintenance of blood calcium concentration is critical, and the total serum calcium level is $2.1-2.6 \mathrm{mmol} / \mathrm{L}$ under normal circumstances (1). Hypercalcemia, defined as a concentration of calcium over $3.5 \mathrm{mmol} / \mathrm{L}$, is a clinical emergency which can cause hypercalcemic crisis and even endanger the patients' life (2).

Hypercalcemia is divided into parathyroid hormone (PTH)-dependent hypercalcemia and non-PTH-dependent hypercalcemia, and PTH is the key index in the diagnosis of hypercalcemia (3). It was reported that the correction of serum calcium and phosphorus levels of non-PTHdependent hypercalcemia were higher than the PTHdependent hypercalcemia. The blood albumin levels of non-
PTH-dependent hypercalcemia were lower than the PTH dependent hypercalcemia. The increase of serum calcium concentration is caused by the redistribution of calcium in bone and the inhibition of parathyroid secretion, which is known as non-parathyroid hypercalcemia (4).

The monitoring of serum calcium and parathyroid hormone is very important in lactating women, which usually affect fetal development and maternal health. And the clinical cases of non-parathyroid hypercalcemia during lactation are rare. This paper reported a case of nonparathyroid hypercalcemia during lactation in order to optimize the diagnosis and treatment of this condition.

We present the following article in accordance with the CARE reporting checklist (available at https://dx.doi. org/10.21037/apm-21-2196). 


\section{Case presentation}

\section{General information}

A 27-year-old female patient was admitted to Wuxi People's Hospital on July 11, 2019 due to "fatigue, anorexia, and pain in both knees for half a month". The patient had a birth history of 1-0-0-1 and was 80 days postpartum. In the recent half a month, the patient had conscious fatigue and discomfort, accompanied by pain in both knees without obvious inducement. At the same time, the patient had decreased food intake. In the past 3 days, the symptoms worsened, accompanied by limb numbness. No pain in other joints and muscles and no obvious movement disorder were reported. The patient had no abdominal pain and distension, no obvious abnormality in urination and defecation, and no nausea, vomiting, or fever. The patient had no smoking and alcohol drinking history. No family history of cancer was reported.

All procedures performed in studies involving human participants were in accordance with the ethical standards of the institutional and/or national research committee(s) and with the Helsinki Declaration (as revised in 2013). Written informed consent was obtained from the patient for publication of this case report and accompanying images. A copy of the written consent is available for review by the editorial office of this journal.

\section{Examination}

The patient's body temperature was $36.5^{\circ} \mathrm{C}$, pulse was 80 times/min, breathing was 15 times/min, and blood pressure was 100/54 mmHg. Her height was $162 \mathrm{~cm}$ and her weight was $40 \mathrm{~kg}$. She had a clear mind, thin body, no Cushing appearance, no bilateral goiter, no tenderness in the sternum, no abnormality in the heart and lungs, no tenderness in the abdomen, no percussion pain in the kidneys, no deformity or tenderness in joints, and normal muscle tension in limbs. The lactation volume of the bilateral breasts was approximately 2,000 mL/24 h.

The patient's blood calcium was $3.45 \mathrm{mmol} / \mathrm{L}$ and blood PTH was $0.2 \mathrm{pg} / \mathrm{mL}$, indicating normal thyroid function. In terms of sex hormones, testosterone was $12.73 \mathrm{ng} / \mathrm{dL}$, estradiol was $9.00 \mathrm{pg} / \mathrm{mL}$, luteinizing hormone was 1.6 U/L, follicle stimulating hormone was $6.72 \mathrm{U} / \mathrm{L}$, and prolactin was $27.91 \mathrm{ng} / \mathrm{mL}$. In terms of growth hormones, her insulin-like growth factor binding protein and insulin-like growth factor I were normal. Her 24-hour urine cortisol was $154.44 \mu \mathrm{g} / 24 \mathrm{~h}$. The immune function examination, rheumatoid examination, and antinuclear antibody examination were normal. There were no significant abnormalities in urinary protein, $\kappa$-light chain, $\lambda$-light chain, and serum protein electrophoresis. In terms of bone metabolic markers, total type I collagen N-terminal lengthening peptide was $188.0 \mathrm{ng} / \mathrm{mL}, \beta$-collagen special sequence was $3,259.0 \mathrm{pg} / \mathrm{mL}, 25$-hydroxyvitamin $\mathrm{D}$ was $21.56 \mathrm{ng} / \mathrm{mL}$, and osteocalcin was $74.54 \mathrm{ng} / \mathrm{mL}$. Her bone mineral density and bone content decreased. No abnormality was found in bone marrow puncture. The whole body bone scan showed no abnormality. Chest and abdominal CT scans showed small nodules in both lungs, ground glass nodules in the left upper lobe of the lung, slightly larger lymph nodes in the left armpit, right kidney stones, and pelvic effusion. Pituitary MRI showed that the pituitary signal was not uniform, locally suspected uplift was observed on the left base, and dynamic enhancement MRI was recommended to exclude the lesions if necessary, dynamic enhancement to exclude lesions. Color Doppler ultrasound of mammary glands showed a change in bilateral mammary glands during lactation, with the possibility of milk deposition.

\section{Diagnosis and treatment}

The patient was diagnosed with hypercalcemia. After admission, the patient was given sufficient rehydration and salmon calcitonin (Novartis Pharmaceuticals). Blood calcium returned to the normal range after treatment, and the disease recurred after the withdrawal of the treatment. One week after admission, the patient was treated with lactation termination therapy, and the blood calcium returned to normal.

\section{Follow-up and outcome}

After discharge, the patient was followed up for 2 weeks via an outpatient visit. Her blood calcium and PTH were normal, and there was no obvious discomfort.

\section{Discussion}

The etiology of hypercalcemia is diverse, and can include the intake of drugs (such as hydrochlorothiazide, lithium, and phosphoric acid), excessive in take of vitamin D, endocrine diseases such as hyperthyroidism, adrenal insufficiency, and acromegaly, abnormal nutritional intake (such as parenteral nutrition of premature infants), and 
lactone syndrome (5). Primary hyperparathyroidism (PHPT) is the most common cause of hypercalcemia, characterized by elevated PTH levels. Therefore, if the PTH level is decreased, further examination is needed to exclude hypercalcemia caused by non-PTH hypercalcemia (6).

When parathyroid secretion is inhibited, the blood calcium concentration increases, referred to as nonparathyroid hypercalcemia. The main reasons are as follows: the redistribution of calcium in bone caused by a malignant tumor; the decrease of intestinal calcium absorption caused by the increase of vitamin $\mathrm{D}$ activity; and the decrease of renal micturition caused by drugs (4). In this case, PTH was significantly decreased, while thyroid function, 25 -hydroxyvitamin $\mathrm{D}$, urinary protein, $\kappa$-light chain, $\lambda$-light chain, serum protein electrophoresis, growth hormones, insulin-like growth factor binding protein, insulin-like growth factor I, adrenocorticotropic hormone (ACTH) rhythm, cortisol rhythm, and 24-hour urinary cortisol were all normal. There were no abnormalities in the examination of immune function, rheumatism, antinuclear antibody, and anti-extractable nuclear antigen (ENA). According to the above examination results, hyperparathyroidism, hyperthyroidism, vitamin D poisoning, adrenal insufficiency, milk alkali syndrome, and other diseases that lead to elevated blood calcium were excluded. In addition, the patient did not take oral thiazide diuretics, a large number of calcium tablets or lithium preparations, and other drugs. Therefore, drug-induced hypercalcemia was also excluded.

Previous studies have indicated that humoral hypercalcemia caused by malignant tumors is usually induced by PTH-related peptide (PTHrP) secreted by the malignant tumor (7-9). PTHrP is a large complex protein synthesized by a variety of tissues, but its chemical properties and functions coincide with PTH (10). The N-terminal part of PTHrP is homologous to PTH, both of which can bind with the PTH type 1 receptor (PTHR) to play a calcium regulatory role, leading to similar bone effects, thus affecting calcium and phosphorus metabolism (11). When humoral hypercalcemia occurs, PTHrP is secreted in large quantities and PTH secretion is inhibited by hypercalcemia, resulting in the decrease of blood PTH level (12). Chest and abdominal CT scans showed small nodules in both lungs, ground glass nodules in the upper lobe of the left lung, and slightly larger lymph nodes in the left armpit. Meanwhile, PET-CT examination in other hospitals showed no obvious abnormalities. Therefore, malignant tumor-induced hypercalcemia was excluded.
Although no evidence indicated that hypercalcemia in this patient was caused by a malignant tumor, the effect of PTHrP cannot be ignored. Studies showed that PTHrP appeared in circulating blood and exhibited endocrine functions in the following 3 cases (13): (I) humoral hypercalcemia: PTHrP derived from tumor cells promotes bone absorption; (II) lactation: PTHrP produced by the mammary gland and released into circulating blood; (III) fetal period: PTHrP manages the placental circulation between the mother and fetus. A previous study (14) found that at the age of 6 months, the average lactation volume of the mother was $769[335-1,144] \mathrm{mL} / 24 \mathrm{~h}$. The amount of lactation of the patient in this case was as high as 2,000 $\mathrm{mL} / 24 \mathrm{~h}$, thus, we speculated that PTHrP may be produced by the breast and released into the blood, leading to hypercalcemia and inhibiting the secretion of PTH.

It was believed that the phenomena of bone loss, calcium loss, and imbalance of bone metabolism in women during lactation were related to the low level of estrogen during lactation and the increased levels of PTHrP and prolactin (15). A previous study found that subcutaneous injection of PTHrP or leuprorelin acetate (long-acting GnRH agonist) into adult female mice can cause bone loss (16). Although the exact mechanism of bone loss during lactation is still unclear, it was shown that the mobilization of calcium during lactation and the resulting bone loss were mostly attributed to the decrease in estrogen level and the increase in PTHrP level secreted by the mammary glands, as both can lead to an increase in the number of osteoclasts (17). Therefore, this patient had active bone metabolism and decreased bone mineral density, which further highlights the correlation between hypercalcemia and PTHrP.

Although breastfeeding osteoporosis may be related to an increase in PTHrP, the specific mechanism of PTHrP leading to breastfeeding osteoporosis is still unclear (18). However, according to the correlation between PTHrP, breastfeeding osteoporosis, and hypercalcemia, we speculated that the treatment of lactation in this patient could reduce lactation and inhibit the secretion of PTHrP in the breast, which might improve hypercalcemia and its related symptoms.

In 2010, a case of non-parathyroid hypercalcemia during lactation with severe osteoporosis was reported. The daily lactation volume of the patient was 2,500-3,000 mL, and the level of PTHrP was significantly increased. After lactation termination therapy, the blood calcium quickly returned to normal, and the PTHrP also 
returned to normal after more than 20 days. In this case, the patient had a large amount of milk excretion, and a lot of calcium was lost from the milk. In order to meet the needs of calcium in milk, excessive hyperplasia of breast tissue and excessive secretion of PTHrP promoted the mobilization of bone calcium, leading to significant hypercalcemia and severe osteoporosis. After the treatment, the loss of calcium in milk was reduced, the stimulation of PTHrP was weakened, the secretion of PTHrP was reduced, the blood calcium returned to normal, and PTH gradually returned to normal (19). This case confirmed our conjecture. In addition, some studies indicated that bone resorption caused by the decrease of estrogen level and the increase of PTHrP level during lactation was due to the increase of prolactin secretion (20). Long-term hyperprolactinemia can affect bone metabolic rate, and when it is associated with hypogonadism, it may lead to a decrease in bone mineral density (21). In the early years of this field, a Chinese study reported that hyperprolactinemia could induce osteoporosis. The 35 patients in this report were all young and middleaged women with hyperprolactinemia. The bone mineral density of the $2^{\text {nd }}-4^{\text {th }}$ lumbar vertebrae decreased, and the levels of osteocalcin and calcitonin decreased (22). Pituitary MRI showed that the left basal part of the pituitary was suspiciously uplifted, but the prolactin and sex hormone levels were normal. Therefore, hyperprolactinemia was excluded, hyperprolactinemia usually leads to massive lactation, then resulting in PTHrP-related symptoms. However, there has been no report on the etiology and specific pathological mechanisms of excessive lactation in female lactation.

In conclusion, this report introduced a rare case of nonparathyroid hypercalcemia during lactation, which suggested that clinicians should consider the correlation between hypercalcemia and lactation after excluding other possible causes. For patients with non-parathyroid hypercalcemia caused by excessive lactation, lactation termination therapy can often achieve curative efficacy. In addition, in order to further treat non-parathyroid hypercalcemia during lactation, it is necessary to further accumulate clinical cases and improve the overall understanding of the pathogenesis of the disease.

\section{Acknowledgments}

Funding: None.

\section{Footnote}

Reporting Checklist: The authors have completed the CARE reporting checklist. Available at https://dx.doi. org/10.21037/apm-21-2196

Conflicts of Interest: All authors have completed the ICMJE uniform disclosure form (available at https://dx.doi. org/10.21037/apm-21-2196). The authors have no conflicts of interest to declare.

Ethical Statement: The authors are accountable for all aspects of the work in ensuring that questions related to the accuracy or integrity of any part of the work are appropriately investigated and resolved. All procedures performed in studies involving human participants were in accordance with the ethical standards of the institutional and/or national research committee(s) and with the Helsinki Declaration (as revised in 2013). Written informed consent was obtained from the patient for publication of this case report and accompanying images. A copy of the written consent is available for review by the editorial office of this journal.

Open Access Statement: This is an Open Access article distributed in accordance with the Creative Commons Attribution-NonCommercial-NoDerivs 4.0 International License (CC BY-NC-ND 4.0), which permits the noncommercial replication and distribution of the article with the strict proviso that no changes or edits are made and the original work is properly cited (including links to both the formal publication through the relevant DOI and the license). See: https://creativecommons.org/licenses/by-nc-nd/4.0/.

\section{References}

1. Zhang YP. Etiology, diagnosis and treatment of hypercalcemia. Journal of Modern Medicine and Health Research 2018;2:194-5.

2. Zhao NR, Su N, Gao F, et al. Etiological analysis of patients with hypercalcemia. Journal of Hebei Medical University 2019;40:1009-16.

3. Zagzag J, Hu MI, Fisher SB, et al. Hypercalcemia and cancer: Differential diagnosis and treatment. CA Cancer J Clin 2018;68:377-86.

4. Bollerslev J, Pretorius M, Heck A. Parathyroid hormone independent hypercalcemia in adults. Best Pract Res Clin 
Endocrinol Metab 2018;32:621-38.

5. Goltzman D. Nonparathyroid Hypercalcemia. Front Horm Res 2019;51:77-90.

6. Liao Q, Zhao YP. Pay attention to the diagnosis and treatment of primary hyperparathyroidism. Chinese Journal of Practical Surgery 2014;34:289-91.

7. Wright LE, Guise TA. The Role of PTHrP in Skeletal Metastases and Hypercalcemia of Malignancy. Clinical Reviews in Bone and Mineral Metabolism 2014;12:119-29.

8. Szymanski JJ, Otrock ZK, Patel KK, et al. Incidence of humoral hypercalcemia of malignancy among hypercalcemic patients with cancer. Clin Chim Acta 2016;453:190-3.

9. Feldenzer KL, Sarno J. Hypercalcemia of Malignancy. J Adv Pract Oncol 2018;9:496-504.

10. Rey E, Jacob CE, Koolian M, et al. Hypercalcemia in pregnancy - a multifaceted challenge: case reports and literature review. Clin Case Rep 2016;4:1001-8.

11. Sutkeviciute I, Clark LJ, White AD, et al. PTH/PTHrP Receptor Signaling, Allostery, and Structures. Trends Endocrinol Metab 2019;30:860-74.

12. Guidelines for the diagnosis and treatment of primary hyperparathyroidism. Chinese Journal of Osteoporosis and Bone Mineral Research 2014;7:187-98.

13. Wang ZY, Sheng Y. Research progress of parathyroid hormone related protein. Guangdong Medical Journal 2013;34:2591-4.

14. Sonawane BR. Chemical contaminants in human milk: an overview. Environ Health Perspect 1995;103 Suppl 6:197-205.

Cite this article as: Shi W, Wang X, Yang J, Xu L, Bu R. Nonparathyroid hypercalcemia during lactation: a case report. Ann Palliat Med 2021;10(8):9304-9308. doi: 10.21037/apm-21-2196
15. Link TM, Majumdar S. Osteoporosis imaging. Radiol Clin North Am 2003;41:813-39.

16. Ardeshirpour L, Brian S, Dann P, et al. Increased PTHrP and decreased estrogens alter bone turnover but do not reproduce the full effects of lactation on the skeleton. Endocrinology 2010;151:5591-601.

17. Lotinun S, Ishihara Y, Nagano K, et al. Cathepsin K-deficient osteocytes prevent lactation-induced bone loss and parathyroid hormone suppression. J Clin Invest 2019;129:3058-71.

18. Koren R, Neeman O, Koren S, et al. Humoral hypercalcemia of pregnancy treated with bisphosphonates. Arch Endocrinol Metab 2018;62:125-8.

19. Gu P, Wang J, Wang YY, et al. A case report of hypercalcemia and severe osteoporosis caused by elevated parathyroid hormone related protein during lactation. Chinese Journal of Endocrinology and Metabolism 2010;26:169-70.

20. Terauchi M. The effects of pregnancy and lactation on bone and mineral metabolism. Clin Calcium 2019;29:35-8.

21. De Hert M, Detraux J, Stubbs B. Relationship between antipsychotic medication, serum prolactin levels and osteoporosis/osteoporotic fractures in patients with schizophrenia: a critical literature review. Expert Opin Drug Saf 2016;15:809-23.

22. Hu SS, Song WB. Amenorrhea galactorrhea syndrome and osteoporosis in young and middle-aged people. Chinese Journal of Osteoporosis 1996;4:59-60.

(English Language Editor: C. Betlzar) 